\title{
Outcomes of Percutaneous Nephrolithotomy with or without Nephrostomy Tube: A Comparative Study
}

\section{Mohammad Ibrahim Ali1 ${ }^{*}$, Prodyut Kumar Saha1, Shafiqul Alam Chowdhury1, Nahid Kamal', Mostafiger Rahman ${ }^{3}$, Sazzad Hossain ${ }^{4}$, Ripan Debnath', Mohammad Saruar Alam', A. K. M. Shahidur Rahman', Kamrul Islam6}

\author{
${ }^{1}$ Department of Urology, Dhaka Medical College Hospital, Dhaka, Bangladesh \\ ${ }^{2}$ District Hospital, Sherpur, Bangladesh \\ ${ }^{3}$ Department of Urology, Prime Medical College, Rangpur, Bangladesh \\ ${ }^{4}$ Apollo Hospital Ltd. Gulshan, Dhaka, Bangladesh \\ ${ }^{5}$ Department of Nephrology, Bangabandhu Sheikh Mujib Medical University (BSMMU), Dhaka, Bangladesh \\ ${ }^{6}$ Zilla Sadar Hospital, Habigong, Bangladesh \\ Email: ^ibrahimurology@gmail.com
}

How to cite this paper: Ali, M.I., Saha, P.K., Chowdhury, S.A., Kamal, N., Rahman, M., Hossain, S., Debnath, R., Alam, M.S., Rahman, A.K.M.S. and Islam, K. (2019) Outcomes of Percutaneous Nephrolithotomy with or without Nephrostomy Tube: A Comparative Study. Journal of Biosciences and Medicines, 7, 52-60. https://doi.org/10.4236/jbm.2019.73006

Received: January 30, 2019

Accepted: March 4, 2019

Published: March 7, 2019

Copyright $\odot 2019$ by author(s) and Scientific Research Publishing Inc. This work is licensed under the Creative Commons Attribution International License (CC BY 4.0).

http://creativecommons.org/licenses/by/4.0/

\begin{abstract}
Objectives: To compare the outcomes of Percutaneous Nephrolithotomy (PCNL) with or without nephrostomy tube. Materials \& Methods: This prospective comparative study intended to compare the outcomes between PCNL without nephrostomy tube and PCNL with nephrostomy tube. A total of 50 cases of renal stone disease planned for PCNL in Dhaka Medical College Hospital from July 2015 to June 2017, were included in this study according to the statistical calculation. Cases were randomly allocated to group $\mathrm{A}$ (PCNL without nephrostomy tube) and group B (PCNL with nephrostomy tube). Each group consisted of 25 patients. The outcome variables were post operative pain, requirement of analgesic, leakage of urine and post operative hospital stay. Data were analyzed and compared by statistical tests. Results: No significant differences were found regarding age $(p=0.95)$, sex $(p=0.55)$ and operation time $(p=0.36)$ between two groups. Post operative pain ( $p<$ $0.0007)$ and analgesic requirement $(p<0.0001)$ were less in group A than group B cases. Leakage of urine is higher in group B in comparison to group A $(p<0.0001)$. Post operative hospital stay is shorter in group A than group B cases which is statistically significant $(p<0.0001)$. Conclusions: Percutaneous nephrolithotomy without nephrostomy tube is better than percutaneous nephrolithotomy with nephrostomy tube in selective cases. It significantly reduces post operativepain, analgesic requirement and postoperative hospital stay. So percutaneous nephrolithotomy without nephrostomy tube is
\end{abstract}


safe and effective.

\section{Keywords}

Nephrostomy, Percutaneous Nephrolithotomy (PCNL), Renal Stone Disease

\section{Introduction}

Urinary calculi are the third most common affliction of the urinary tract, exceeded only by urinary tract infections and pathologic conditions of the prostate [1]. The lifetime prevalence of kidney stone disease is estimated at $1 \%$ to $15 \%$, with the probability of having a stone varying according to age, gender, race, and geographic location [2]. The primary goal of surgical stone management is to achieve maximal stone clearance with minimal morbidity to the patient. Percutaneous nephrolithotomy (PCNL) is currently the preferred first-line treatment and the operation of choice for the management of large, complex renal calculi. Its high success rate, low morbidity and complication rates has replaced the open surgical approach [3].

The standard PCNL procedure consists of percutaneous access to the kidney and the formation of a working tract connecting the flank surface with the renal collecting system to allow endoscopic stone fragmentation and removal. A temporary nephrostomy tube is usually left in place at the end of the procedure to tamponade bleeding, and allows drainage and delayed second-look nephroscopy [3].

Technological advancements and refinements have contributed to further lowering the morbidity associated with this procedure. Such refinements include the use of a smaller working sheath and smaller nephroscope (mini PCNL), sealing of the percutaneous access track with haemostatic agents, substituting general anesthesia with regional blocks (ambulatory spinal PCNL), avoidance of a nephrostomy tube (tubeless PCNL) and avoidance of both nephrostomy tube and JJ stent (totally tubeless) [4].

Although nephrostomy tube plays an essential role in PCNL, the potential complications related to its use should not be underestimated. However, placing nephrostomy tube is clearly associated with increased morbidity such as more pain, tract site leakage of urine and longer post operative hospital stay [5].

Many authors highlighted the compelling advantages of the tubeless approach. PCNL has been practiced successfully in many centers in Bangladesh since 2000. Most of the urologists have been giving nephrostomy tube before ending the procedure. Recently tubeless PCNL are practicing in selective cases with good result.

For patients with normal preoperative renal function, uncomplicated PCNL, minimum bleeding, and complete clearance of stones, nephrostomy tube may not be required. There were several studies on PCNL without nephrostomytube. Tubeless PCNL was done by Gupta V. et al. (2004) [6] and found a good result. 
Multiple comparative studies have been shown that postoperative outcome of PCNL without nephrostomy tube is better than the PCNL with nephrostomy tube. The aim of the study was to compare the outcome of PCNL with or without nephrostomy tube.

\section{Materials and Methods}

This prospective comparative study was carried out among the patients with renal stone planned for PCNL in the Department of Urology, Dhaka Medical College Hospital, Dhaka, Bangladesh during the period of July 2015 to June 2017. The selection criteria were age 18 - 50 years, single stone, stone size 2 - $3 \mathrm{~cm}$, single subcostal puncture, preoperative normal renal function and negative urine culture. Patients having an anatomical anomaly of the kidney, incomplete stone clearance, multiple caliceal punctures, acomplicated procedure such as perforation or tear in the pelvicalyceal system, excessive bleeding, injury to the adjacentorgan were excluded from the study.

A total of 50 patients were selected as cases by statistical formula and randomly allocated to two groups. Group A consisted of 25 patients who underwent PCNL without nephrostomy tube. Group B consisted of 25 patients who underwent PCNL with nephrostomy tube. All the odd-numbered cases were allocated for group A; the even-numbered cases were allocated for group B.

This study was approved by Ethical Review Committee, Dhaka Medical College, Dhaka, Bangladesh. Written informed consent was taken from every case. Each study subject was evaluated by history, physical examination and investigation. History was taken regarding previous renal surgery, urinary tract infection, bronchial asthma, diabetes mellitus, hypertension and ischemic heart disease. Physical examination included general examination and examination of the renal region and other parts of genitourinary system. All patients were investigated properly by urine for routine and microscopic examination and culture sensitivity, serum creatinine, ultrasonography. Intravenous urogram or CT urogram was done to see the anatomy of the pelvicalyceal system and the location of the stone, any obstruction in theuretero-pelvicjunction (UPJ), ureter and vesico-ureteric junction (VUJ). Other routine investigations were done as per protocol.

Standard operative procedure was followed in every case. Under general anesthesia, the patient in lithotomy position, an open-ended 6 Fr ureteric catheter passed up to the kidney by urethra-cystoscopy allowing introducing the contrast materials to opacify and distending the collecting system. Once the ureteral catheter was inserted, the patient was placed in a prone position. Contrast material was instilled through the ureteral catheter foropacification of the collecting system. Percutaneous access was created under fluoroscopic guidance using $18 \mathrm{G}$ access needle into the targetedcalyx. A 0.035 inch floppy tipped guide wire was inserted into the needle and advanced into the calyceal system either to ureter or coiled within the renal pelvis. Access needle was removed and the skin was incised. The nephrostomy tract was dilated with Alken coaxial metallic dilators up 
to $24 \mathrm{Fr}$ and a $26 \mathrm{Fr}$ Amplatz sheath positioned into the renal collecting systems. The stone was disintegrated using pneumatic lithotripsy. Complete clearance was confirmed by fluoroscopy and nephroscopy. After completion of operation nephrostomy tube was not placed in group-A cases and nephrostomy tube was placed in group-B cases. Double J (DJ) stent was placed in all cases.

During postoperative period, both groups were compared regarding pain, analgesic requirement, leakage of urine and length of postoperative hospital stay.

Post operative pain was estimated by visual analog scale (VAS) 6 hours after the operation. After completion of procedure $1^{\text {st }}$ dose of injectionpethidine $(1 \mathrm{mg} / \mathrm{kg}$ body-weight, intramuscular) was given in every case and further doses were given as per need.

Urine leakage was estimated by changing the dressing at the percutaneous access site every 4 hours and duration of the urinary leak was determined after the overlying dressing was completely dry for 4 hours. The urine leakage of group B was estimated by the same method after removal of nephrostomy tube.

Postoperative hospital stay was calculated from recovery of the patient from anesthesia after the operation to discharge from the hospital.

Data were processed manually by Microsoft office 2007 and analyzed by online graphpad calculator and SPSS (Statistical package for social sciences) Version 22.0. Quantitative data were expressed as mean and standard deviation and comparison were done by student " $t$ " test. Qualitative data were expressed as frequency, percentage and comparisons were carried out by Chi-square $\left(\mathrm{X}^{2}\right)$ test. A probability value $(p)$ of less than 0.05 was considered to indicate statistical significance. The summarized findings were then presented in tables and graphs.

\section{Results}

A total of 50 cases of renal stone disease were allocated to group A (PCNL without nephrostomy tube) and group B (PCNL with nephrostomy tube). The outcome variables were post operative pain, analgesic requirement, urine leakage, post operative hospital stay.

\subsection{Age Distribution}

The mean age of group A was $44.04( \pm 10.97)$ years and that of group B was 44.24 $( \pm 12.75)$ years. The age differences between the two groups were not statistically significant $(p=0.95)$ (Table 1$)$.

\subsection{Sex Distribution}

In this study $72 \%$ (18) cases in group-A and 64\% (16) cases in group-B were male. On the other hand $28 \%$ (7) cases in group A and $36 \%$ (9) cases of group B were female (Figure 1). The sex distribution between the two groups was not statistically significant $(p=0.55)$. Data were analyzed using Chi-square test. Level of significance was $<0.05$. 


\subsection{Operation Time}

The difference of mean operation time in group A $(79.24 \pm 21.50)$ and group B $(84.64 \pm 20.09)$ was notstatistically significant $(p=0.36)$ (Table 2$)$.

\subsection{Post Operative Pain}

Post operative pain was compared by Visual Analog Scale (VAS). The mean pain intensity of group A was $20.00 \pm 20.95 \mathrm{~mm}$ and that of group Bwas $41.88 \pm 21.82$ $\mathrm{mm}$. There was a significant difference of pain intensity in between two groups $(p<0.0007)$ (Table 3).

\subsection{Analgesic Requirement}

The mean dose of pethidinerequired in group A and group B was $66 \pm 14.21 \mathrm{mg}$ and $120 \pm 27.95 \mathrm{mg}$ respectively. The analgesic requirement of PCNL without nephrostomy tube group was significantly lower than the PCNL with nephrostomy tube group $(p<0.0001)$ (Table 4$)$.

\subsection{Leakage of Urine}

The mean leakage of urine through the puncture site in group A cases was $7.68 \pm$ 4.88 hours and in group B cases was $16.16 \pm 6.05$ hours. The difference of leakage of urine between the two groups was statistically significant $(p<0.0001)$ (Table 5).

\subsection{Postoperative Hospital Stay}

The mean duration of post operative hospital stay in group A was $1.92 \pm 0.49$ days and in group B was $2.84 \pm 0.68$ days. The postoperative hospital stay in PCNL without nephrostomy tube group was shorter than PCNL with nephrostomy tube group $(p<0.0001)$ (Table 6$)$.

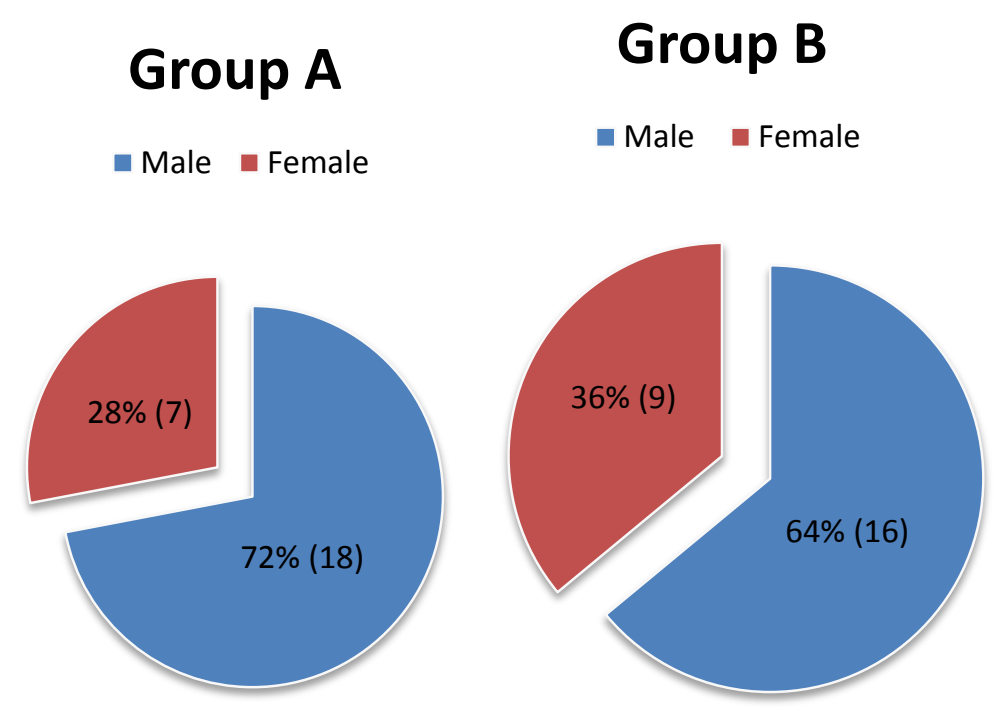

Figure 1. Pie Chart showing thedistribution of patients by sex. 
Table 1. Distribution of patients according to age between two groups.

\begin{tabular}{cccc}
\hline \multirow{2}{*}{ Age } & \multicolumn{3}{c}{ Group } \\
\cline { 2 - 4 } & $\begin{array}{c}\text { Group A } \\
(\mathrm{n}=25)\end{array}$ & $\begin{array}{c}\text { Group B } \\
(\mathrm{n}=25)\end{array}$ & $p$-value \\
\hline Mean $\pm \mathrm{SD}$ & $44.04 \pm 10.97$ & $44.24 \pm 12.71$ & 0.95 \\
\hline
\end{tabular}

Data were analyzed using Student's t-test and level of significance was $<0.05$.

Table 2. Comparison of operation time between two groups.

\begin{tabular}{cccc}
\hline \multirow{2}{*}{$\begin{array}{c}\text { Operation time } \\
\text { (minutes) }\end{array}$} & $\begin{array}{c}\text { Group A } \\
(\mathrm{n}=25)\end{array}$ & $\begin{array}{c}\text { Group B } \\
(\mathrm{n}=25)\end{array}$ & $p$-value \\
\cline { 2 - 4 } & $79.24 \pm 21.50$ & $84.64 \pm 20.09$ & 0.36 \\
\hline
\end{tabular}

Data were analyzed using Student's t-test and level of significance was $<0.05$.

Table 3. Comparison of postoperative pain between two groups.

\begin{tabular}{|c|c|c|c|}
\hline \multirow[b]{2}{*}{$\begin{array}{c}\text { Visual Analog } \\
\text { Scale (mm) }\end{array}$} & \multicolumn{2}{|c|}{ Group } & \multirow[b]{2}{*}{$p$-value } \\
\hline & $\begin{array}{c}\text { Group A } \\
\text { (PCNL without nephrostomy tube) } \\
(\mathrm{n}=25)\end{array}$ & $\begin{array}{c}\text { Group B } \\
\text { (PCNL with nephrostomy tube) } \\
(\mathrm{n}=25)\end{array}$ & \\
\hline Mean \pm SD & $20.00 \pm 20.95$ & $41.88 \pm 21.82$ & $<0.0007$ \\
\hline
\end{tabular}

Data were analyzed using Student's t-test and level of significance was $<0.05$.

Table 4. Comparison of postoperative analgesic requirement betweentwo groups.

\begin{tabular}{|c|c|c|c|}
\hline \multirow{2}{*}{$\begin{array}{c}\text { Analgesics requirement } \\
\text { (mgofpethidine) }\end{array}$} & \multicolumn{2}{|c|}{ Group } & \multirow[b]{2}{*}{$p$-value } \\
\hline & $\begin{array}{c}\text { Group A } \\
(\mathrm{n}=25)\end{array}$ & $\begin{array}{l}\text { Group B } \\
(\mathrm{n}=25)\end{array}$ & \\
\hline Mean \pm SD & $66 \pm 14.21$ & $120 \pm 27.95$ & $<0.0001$ \\
\hline
\end{tabular}

Data were analyzed using Student's t-test and level of significance was $<0.05$.

Table 5. Comparison of leakage of urine between two groups.

\begin{tabular}{|c|c|c|c|}
\hline \multirow[b]{2}{*}{$\begin{array}{l}\text { Leakage of urine } \\
\text { (hour) }\end{array}$} & \multicolumn{2}{|l|}{ Group } & \multirow[b]{2}{*}{$p$-value } \\
\hline & $\begin{array}{c}\text { Group A } \\
\text { (PCNL without nephrostomy tube) } \\
(\mathrm{n}=25)\end{array}$ & $\begin{array}{c}\text { Group B } \\
\text { (PCNL with Nephrostomy) } \\
(\mathrm{n}=25)\end{array}$ & \\
\hline Mean \pm SD & $7.68 \pm 4.88$ & $16.16 \pm 6.05$ & $<0.0001$ \\
\hline
\end{tabular}

Data were analyzed using Student's t-test and level of significance was $<0.05$.

Table 6. Comparison of postoperative hospital stay between two groups.

\begin{tabular}{cccc}
\hline \multirow{2}{*}{$\begin{array}{c}\text { Postoperative hospital } \\
\text { stay (day) }\end{array}$} & $\begin{array}{c}\text { Group } \\
(\mathrm{n}=25)\end{array}$ & $\begin{array}{l}\text { Group B } \\
(\mathrm{n}=25)\end{array}$ & $p$-value \\
\cline { 2 - 3 } & $1.92 \pm 0.49$ & $2.84 \pm 0.68$ & $<0.0001$ \\
\hline
\end{tabular}

Data were analyzed using Student's t-test and level of significance was $<0.05$. 


\section{Discussion}

In standard PCNL, a temporary nephrostomy tube is usually left in place at the end of the procedure to tamponade of bleeding, allow urinary drainage and delayed second-look nephroscopy if needed. Currently in uncomplicated PCNL nephrostomy tube may not be required.

The present study has been designed to compare the outcome of PCNL with or without nephrostomy tube for the management of renal stone disease. Cases were selected by inclusion and exclusion criteria. Patients with renal stone planned for PCNL were divided into two groups. Patients belonging group A were treated by PCNL without nephrostomy tube and patients belonging group B were treated by PCNL with nephrostomy tube.

Results of treatment of both groups were compiled and analyzed. Age, sex, operation time, post operative pain, post operative analgesic requirement, leakage of urine and post operative hospital stay were compared between two groups.

The age range of the patients in this study was between 18 years and 50 years. The mean age of the patients of group A and group B were $41.68 \pm 8.89$ years and $41.6 \pm 9.79$ years respectively. The age range of the present study was more or less comparable with the study done by Bellman G.C. et al. (1997) [7] to evaluate the role of routine placement of nephrostomy tube following percutaneous surgery on 50 patients. A Study was done by Aghamir et al. (2004) [8] and Gupta et al. (2004) [6] on tubeless and stentless PCNL where the age range more or less comparable with the present study.

In this study $72 \%$ cases in group-A and about $64 \%$ cases in group-B were male. On the other hand $28 \%$ cases in group A and $36 \%$ cases of group B were female. The gender distribution between two groups was not statistically significant ( $p=0.55)$. Moosanejad et al. (2016) [9] was done a randomized clinical trial comparing totally tubeless PCNL and standard PCNL group. The gender difference of two groups was also statistically insignificant $(p=0.17)$. So there was a similarity between to study regarding the gender of cases.

The mean operating time in this study was $79.24 \pm 21.5 \mathrm{~min}$ in PCNL without nephrostomy tube and $84.64 \pm 20.09 \mathrm{~min}$ in PCNL with nephrostomy tube. The difference of operation time between the groups was not significant $(p=0.363)$. Desai et al., (2004) [10] have done a study on PCNL with large bore nephrostomy tube, small bore nephrostomy tube and tubeless group where the mean minutes of operation time were $44.5 \pm 13.2,45 \pm 11.7$ and $45 \pm 13.7$ in large bore nephrostomy tube, small bore nephrostomy tube and tubeless group respectively. There were no differences between groups regarding operation time. In a study of Aghamir et al. (2004) [8], a total of 43 patients underwent totally tubeless PCNL where the operating time did not differ significantly between groups. In this study the stone clearance rate was $100 \%$. Karamiand Gholamrezaie (2004) [11] showed stone clearance rate of a tubleless PCNL was $90 \%$.

PCNL without nephrostomy tube was associated with less post operative pain (VAS $=20.00 \pm 20.95 \mathrm{~mm})$ in comparison to PCNL with nephrostomy tube 
$(\mathrm{VAS}=41.88 \pm 21.82 \mathrm{~mm})$. It is statistically significant $(p<0.0007)$. Hemendra et al. (2008) [12] conducted a study of tubeless PCNL of 454 patients. They showed that tubeless PCNL associated with less post operative pain in comparison to standard PCNL $(p<0.001)$ and it was similar to the present study.

This study showed that PCNL without nephrostomy tube requires less dose of analgesic (66 $\pm 14.21 \mathrm{mg}$ pethidine) in comparison to PCNL with nephrostomy tube $(120 \pm 27.95 \mathrm{mg}$ pethidine $)$ which was statistically significant $(p=0.001)$. Moosanejad et al. (2016) [8] compared totally tubeless PCNL and standard PCNL for kidney stones and showed that the dose of pethidine was significantly less in tubeless PCNL group than for standard PCNL group $(p=0.003)$.

In the present study mean percutaneous tract site urine leakage was $7.68 \pm$ 4.88 hours in group A and $16.16 \pm 6.05$ hours in group B, which was statistically significant $(p=0001)$. The tubeless PCNL group was associated with the shortest duration of postoperative percutaneous tract site leakage of urine. Desai et al. (2004) [10] studied that tubeless PCNL had the shortest duration (4.8 hours) of percutaneous tract site urine leak compared to PCNL with nephrostomy tube (21.4 hours) $(p<0.05)$. Although leakage of urine usually resolves spontaneously, it can often be bothersome to the patients when persists for a longer time.

Patients underwent the PCNL without nephrostomy tubehadashorter postoperative hospital stay in comparison to PCNL with nephrostomy tube. In this study postoperative hospital stay was $1.92 \pm 0.49$ days for the patient of PCNL without nephrostomy tube and $2.84 \pm 0.68$ days for PCNL with nephrostomy tube. The result was statistically significant $(p=0.0001)$. The studies of Lojanapiwatv et al. (2001) [13]; Aghamir et al. (2004) [8] and Gupta et al. (2004) [6] on PCNL without nephrostomy tube and JJ stent compared with standard PCNL revealed significantly shorter hospital stay for PCNL without nephrostomy tube.

\section{Conclusion}

Percutaneous nephrolithotomy without nephrostomy tube (tubeless PCNL) is safe, and effective in selective cases with stone size $2-3 \mathrm{~cm}$, normal pre-operative renal function, single percutaneous puncture, minimum bleeding, no injury of the collecting system, no obstruction and complete stone clearance. It significantly reduces post operative pain, analgesic requirement, leakage of urine and postoperative hospital stay. Sopercutaneous nephrolithotomy without nephrostomy tube is safe and effective.

\section{Limitations of the Study}

There were several limitations of this study. It was a single centre study with relative small sample size and all complications of PCNL were not included in the study.

\section{Conflicts of Interest}

The authors declare no conflicts of interest regarding the publication of this paper. 


\section{References}

[1] Stoller, ML. (2013) Urinary Stone Disease. In: McAninch, J.W. and Lue, T.F., Eds., Smith \& Tanagho's General Urology, 18th Edition, McGraw-Hill, New York, 249-272.

[2] Margaret, S., Pearle, MD., Jodi, A., Antonelli, M.D. and Yair, L. (2016) Urinary Lithiasis: Etiology, Epidemiology, and Pathogenesis. In: Wein, A.J., Kavoussi, L.R., Partin, A.W. and Peters, C.A., Eds., Campbell-Walsh Urology, 11st Edition, Elsevier Saunders, Philadelphia, 1170-1199

[3] Sofer, M., Lidawi, G., Keren-Paz, G., Yehiely, R., Beri, A. and Matzkin, H. (2010) Tubeless Percutaneous Nephrolithotomy: First 200 Cases in Israel. Israelmedical Association Journal, 12, 164-167.

[4] Yun, S.I., Lee, Y.H., Kim, J.S., Cho, S.R., Kim, B.S. and Kwon, J.B. (2012) Comparative Study between Standard and Totally Tubeless Percutaneous Nephrolithotomy. Korean Journal of Urology, 53, 785-789. https://www.ncbi.nlm.nih.gov/pmc/articles/PMC3502738/

[5] Crook, T.J., Lockyer, C.R., Keoghane, S.R. and Walmsley, B.H. (2008) A Randomized Controlled Trial of Nephrostomy Placement versus Tubeless Percutaneous Nephrolithotomy. The Journal of Urology, 180, 612-614. https://doi.org/10.1016/j.juro.2008.04.020

[6] Gupta, V., Sadasukhi, T.C., Sharma, K.K., Yadav, R.G. and Mathur, R. (2005) Tubeless and Stentless Percutaneous Nephrolithotomy. BJU International, 95, 905-906. https://doi.org/10.1111/j.1464-410X.2005.05432.x

[7] Bellman, G.C., Davidoff, R., Candela, J., Gerspach, J., Kurtz, S. and Stout, L. (1997) Tubeless Percutaneous Renal Surgery. The Journal of Urology, 157, 1578-1582.

[8] Aghamir, S., Hosseini, S. and Gooran, S. (2004) Totally Tubeless Percutaneous Nephrolithotomy. Journal of Endourology, 18, 647-648. https://doi.org/10.1089/end.2004.18.647

[9] Moosanejad, N., Firouzian, A., Hashemi, S.A., Bahari, M. and Fazli, M. (2016) Comparison of Totally Tubeless Percutaneous Nephrolithotomy and Standard Percutaneous Nephrolithotomy for Kidney Stones: A Randomized, Clinical Trial. Brazilian Journal of Medical and Biological Research, 49, 1-6. https://doi.org/10.1590/1414-431X20154878

[10] Desai, M.R., Kukreja, R.A., Desai, M.M., Mhaskar, S.S., Wani, K.A., Patel, S.H. and Bapat, S.D. (2004) A Prospective Randomized Comparison of Type of Nephrostomy Drainage Following Percutaneous Nephrostolithotomy: Large Bore versus Small Bore versus Tubeless. The Journal of Urology, 172, 565-567. https://doi.org/10.1097/01.ju.0000130752.97414.c8

[11] Karami, H. and Gholamrezaie, H.Z. (2004) Totally Tubeless Percutaneous Nephrolithiasis in Selected Patients. Journal of Endourology, 18, 475-479. https://doi.org/10.1089/0892779041271580

[12] Hemendra, S., Amit, K., Hiren, S., Shabbir, K., Sunil, H. and Manish, B. (2008) Tubeless Percutaneous Nephrolithotomy: 3 Years of Experience with 454 Patients. BJU International, 104, 840-846

[13] Lojanapinat, B., Soonthoruphan, S. and Wudhikarns, S. (2001) Tubeless Percutaneous Nephrolithomy in Selected Patients. Journal of Endourology, 15, 711-713. https://doi.org/10.1089/08927790152596299 